ARTICLE

\title{
Longer treatment intervals are associated with reduced treatment persistence in neovascular age related macular degeneration
}

\author{
Kelvin Y. C. Teo (iD ${ }^{1,2,3 凶}$, Vuong Nguyen ${ }^{3}$, Louise O'Toole $\mathbb{D i D}^{4}$, Vincent Daien ${ }^{5}$, Jorge Sanchez-Monroy ${ }^{6}$, Federico Ricci ${ }^{7}$, \\ Theodorus Leonardus Ponsioen (iD ${ }^{8}$, Helena Brosa Morros (iD ${ }^{9}$, Chui Ming Gemmy Cheung ${ }^{1}$, Jennifer J. Arnold ${ }^{10}$, \\ Daniel Barthelmes ${ }^{3,11}$ and Mark C. Gillies ${ }^{3}$
}

(c) The Author(s), under exclusive licence to The Royal College of Ophthalmologists 2022

\begin{abstract}
AIMS: To test the hypothesis that patients treated for neovascular age related macular degeneration (nAMD) with longer treatment intervals are more likely to persist with treatment.

METHODS: Data were obtained from the prospectively-defined Fight Retinal Blindness! registry. Treatment interval at 2 years was stratified based on the mean treatment interval over the three visits prior to and including the 2-year visit. Rates of non-persistence to follow-up were assessed from 2 to 5 years.

RESULTS: Data from 1538 eyes were included. The overall rate of non-persistence was $51 \%$ at 5 years. Patients on longer treatment intervals (12-weeks) at 2 years were found to be less persistent to long-term follow-up. These eyes were found to have fewer active disease visits in the first 2 years $(40 \%)$ than eyes treated at 4 -weekly intervals $(66 \%, p<0.001)$. In the multivariable analysis, better vision at 2 years was associated with a lower risk of non-persistence (hazards ratio [HR] [95\% Cl]: 0.95 [0.93, 0.97], P < 0.001), while longer treatment intervals (HR [95\% Cl]: $1.31[0.95,1.8]$ and $1.54[1.15,2.06]$ for 12-week and > 12-week intervals vs. 4-week intervals, respectively, $P=0.002$ ) and older patients (HR [95\% Cl]: $1.03[1.02,1.04], p<0.001)$ were at higher risk of non-persistence. CONCLUSIONS: We found that patients on longer treatment intervals at 2 years were more likely to be non-persistent with treatment in later years. Reinforcing the need for ongoing treatment is important for patients on longer intervals who may feel complacent or that treatment is no longer effective, particularly if newer, longer lasting agents become widely available.
\end{abstract}

Eye (2023) 37:467-473; https://doi.org/10.1038/s41433-022-01957-z

\section{INTRODUCTION}

Outcomes for patients treated for neovascular age-related macular degeneration (nAMD) with vascular endothelial growth factor (VEGF) inhibitors in clinical practice are consistently worse than those treated in registrational randomized clinical trials (RCT) [1-4]. Nonadherence (defined as not following the prescribed treatment regimen, for example, deviation from planned re-treatment intervals), and non-persistence (defined as complete withdrawal from treatment) are major causes for the discrepancy in treatment outcomes between clinical practice and RCTs $[5,6]$.

The high treatment burden of the frequent, fixed dosing imposed by RCTs are one possible cause of non-adherence or nonpersistence [6]. It is, however, unequivocal that frequent and regular treatments result in better outcomes, especially in cases that have persistent disease activity despite treatment [7-11]. Recognizing the high treatment burden for the patient and healthcare system, several less intensive, variable treatment strategies have evolved that can produce outcomes comparable to fixed treatment regimens [8, 10, 12-14].

Patients treated under a treat-and-extend (TAE) regimen have variable treatment intervals based on disease activity, with shorter intervals for highly active disease and vice versa. It is plausible that patients with more active disease receiving more frequent treatment may suffer from "treatment fatigue" resulting in non-persistence later. Alternatively, patients receiving frequent treatment may be more likely to persist as the benefits of treatment for controlling the disease activity are much more apparent. Here we have studied the association between the treatment interval and injection frequency at 24-months and the 5-year non-persistence to follow-up.

\section{METHODS}

Data were obtained from the Fight Retinal Blindness (FRB!) Registry cohort which prospectively tracks treatment outcomes of nAMD in clinical

\footnotetext{
${ }^{1}$ Singapore Eye Research Institute, Singapore National Eye Centre, Singapore, Singapore. ${ }^{2}$ Duke-NUS Medical School, National University of Singapore, Singapore, Singapore. ${ }^{3}$ The University of Sydney, Save Sight Institute, Discipline of Ophthalmology, Sydney Medical School, Sydney, NSW, Australia. ${ }^{4}$ Mater Private Hospital, Dublin, Ireland. ${ }^{5}$ Department of Ophthalmology, Gui De Chauliac Hospitla, Montpellier, France. ${ }^{6}$ Department of Ophthalmology, Miguel Servet University Hospital, Zaragoza, Spain. ${ }^{7}$ University of Rome Tor

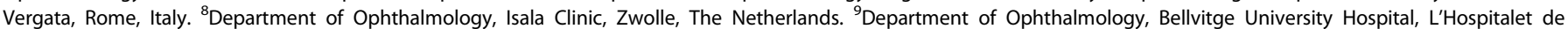
Llobregat, Barcelona, Spain. ${ }^{10}$ Marsden Eye Specialists, Sydney, NSW, Australia. ${ }^{11}$ University Hospital Zurich and University of Zurich, Zurich, Switzerland.

email: kelvin.teo.y.c@singhealth.com.sg
}

Received: 6 September 2021 Revised: 22 December 2021 Accepted: 20 January 2022

Published online: 9 February 2022 
practice [15]. This was a multisite study and included consented patients from Australia, France, Ireland, Italy, Netherlands, New Zealand, Singapore, Spain, and Switzerland. Institutional ethics approval was obtained from each of these country sites; the Human Research Ethics Committees of the University of Sydney, the Royal Victorian Eye, and Ear Hospital, the Royal Australian and New Zealand College of Ophthalmologists, the French Institutional Review Board (IRB) (Société Française d'Ophtalmologie IRB), the Mater Private Hospital IRB, the IRCCS Cà Granda Foundation Maggiore Policlinico Hospital Milan, Singhealth, Singapore the Clinical Research Ethics Committee of Aragon, Spain, and the Cantonal Ethics Committee Zurich, Switzerland. The research described adhered to the tenets of the Declaration of Helsinki.

\section{Registry variables}

The detailed methodology of the FRB! registry has previously been published [15]. Briefly, the registry collects data from every clinical visit. Parameters collected and analyzed for the purpose of this analysis include visual acuity (VA) (expressed as the number of letters read on a logarithm of the minimum angle of resolution (logMAR) VA chart and recorded whichever reading was best: uncorrected, corrected, or pinhole), lesion subtype, lesion activity, treatments administered and interval between treatments.

\section{Clinical characteristics}

Different nAMD subtypes and disease activity were determined by the treating physician using all available imaging tools alone or in combination. These include clinical examination, fluorescein or indocyanine green angiography, and optical coherence tomography (OCT). This reflects the real-world nature of these data.

Lesion subtypes were classified by the treating physician into type 1, 2 , or 3 macular neovascularization (MNV) or others. If the lesion subtype was ambiguous, an "unknown" grading was used. Lesion activity was graded as "active" or "inactive" at each visit. All physicians entering data into the FRB! registry agreed with the following statement: 'Lesions were graded as active if there were features such as sub- or intra-retinal fluid, or new haemorrhage, that suggested that the MNV lesion was active' [16].

\section{Disease management}

Treating physicians determined all management decisions in consultation with the patient including frequency of visits, intended treatment posology, and agent type.

\section{Inclusion and selection criteria}

We included eyes that were treatment-naïve at baseline (the visit of the first injection) initiating VEGF inhibitor monotherapy from 1st January 2013 to 31st December 2015. This allowed for the possibility of completing 5 years of follow-up after their first injection. All eyes had to have at received a minimum of 4 injections and treated for at least 2 years (730 days). Eyes with large gaps of more than 365 days between treatments were excluded.

\section{Patient groups and definitions}

The baseline visit was defined as the visit of the first injection. As most patients in this cohort were treated with a TAE regimen, treatment burden was determined by the average of 3 treatment interval at the 2 year point: 4-week interval (0-34 days), 6-week interval (35-48 days), 8-week interval (49-62 days), 10-week interval (63-76 days), 12-week interval (77-90 days) and $>12$-week interval (90-365 days). The two-year time point was used to define treatment interval groups as this allowed sufficient time for patients under a treat and extend regimen to settle on a regular treatment interval.

A secondary sensitivity analysis was performed where patients were grouped according to increasing treatment frequency in the first 24 months of treatment: 4-8 treatments, 9-13 treatments, 14-18 treatments, $19-23$ treatments, and 23-28 treatments.

\section{Outcomes}

The main outcome was the proportion of patients that were non-persistent to follow-up from 2 to 5 years. Non-persistence was defined as patients that discontinued treatment of their own choice (if formally recorded in the registry) or those who did not have a visit recorded within the last 6 months of data extraction. Otherwise, patients that were formally discontinued within the FRB! registry using one of the following reasons provided (deceased, further treatment futile, medically contraindicated, patient goes to another doctor, or treatment successful) were censored but considered as persisting with treatment. Secondary outcomes included the visual outcomes at 2 years, and the proportion of visits in which the lesion was graded as active.

\section{Statistical Analysis}

Data were summarised using the mean, standard deviation (SD), median, $25^{\text {th }}$ and $75^{\text {th }}$ percentiles $(\mathrm{Q} 1, \mathrm{Q} 3)$, and percentages as appropriate. Baseline characteristics were compared between groups using ANOVA, Kruskal-Wallis, Chi-square or Fisher's exact tests where appropriate.

The proportion of non-persistent eyes was analysed using Kaplan-Meier survival curves with log-rank tests to compare survival curves. Hazards ratios for the risk of non-persistence were calculated using Cox proportional hazards regression analysis with the main predictors being the treatment interval or treatment frequency group, gender, age, VA at 2 years, injection type at 2 years, and lesion type (fixed effects), with adjustment for nesting of outcomes within practice and eyes from the same patient (random effects).

A p-value of less than 0.05 was considered statistically significant. All analyses were conducted using $R$ version 4.0 .5 with the survival package $(V$ 3.2-10) for Kaplan-Meier survival curves and coxme package (V 2.2-16) for Cox proportional hazards models.

\section{RESULTS}

\section{Baseline characteristics}

There were a total of 1538 eyes from 1414 patients eligible for the present analysis (Fig. 1) with most eyes treated at 8-week intervals ( $n=307$, median [Q1, Q3] average interval $=63[46,84]$ days). The baseline visit was defined as the first treatment commenced and recorded in the FRB registry. These baseline characteristics are summarized in Table 1. Eyes treated at 4-week intervals were younger (mean 77.4 years) compared with the other treatment interval groups (mean age ranging from 78.8 to 79.8 years; global $P=0.04$ ). Mean baseline visual acuity was not significantly different between groups with the lowest baseline VA in eyes

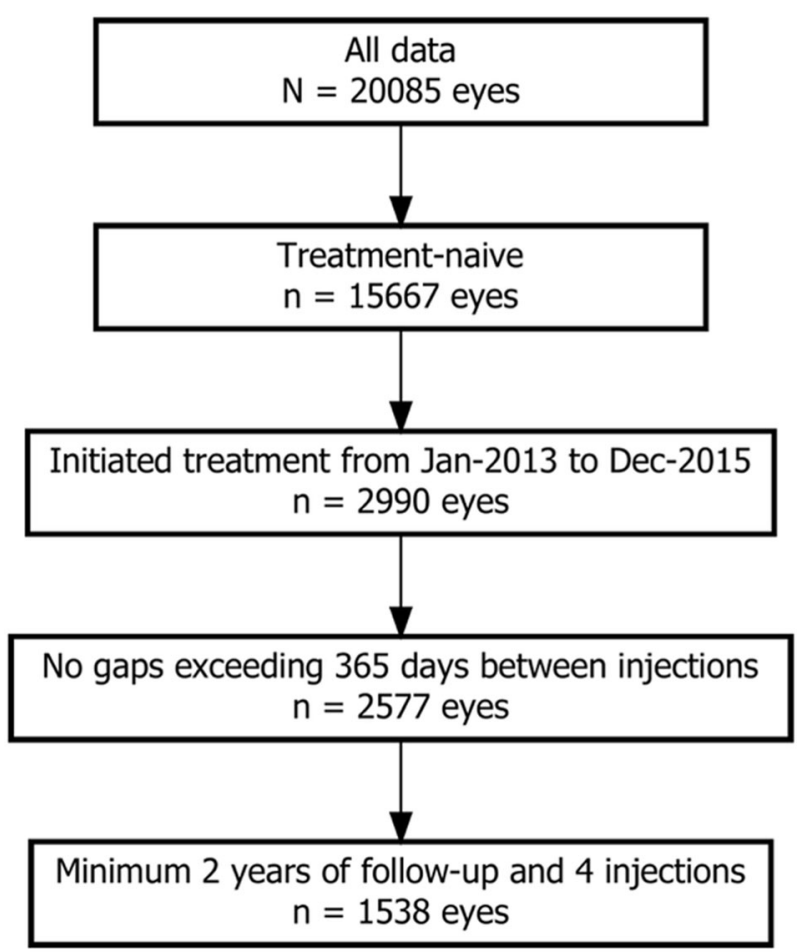

Fig. 1 Selection criteria for inclusion to this study. Flowchart showing the number of eyes remaining following each inclusion or exclusion criteria. 
Table 1. Characteristics of eligible eyes at baseline and at 2 years, grouped by the average treatment interval over the three visits prior to and including the 2-year visit.

\begin{tabular}{|c|c|c|c|c|c|c|c|}
\hline & Eligible cohort & 4-Weeks & 6-Weeks & 8-Weeks & 10-Weeks & 12-Weeks & $>$ 12-Weeks \\
\hline Eyes & 1538 & 160 & 254 & 307 & 262 & 250 & 305 \\
\hline Patients & 1496 & 156 & 249 & 296 & 256 & 241 & 298 \\
\hline Gender, \% female & $62 \%$ & $63 \%$ & $64 \%$ & $62 \%$ & $61 \%$ & $64 \%$ & $63 \%$ \\
\hline Age, mean (SD) & $79.2(8.4)$ & $77.4(8)$ & $79.1(7.6)$ & $78.8(8.7)$ & $79.6(7.6)$ & $79.8(9.6)$ & $79.7(8.3)$ \\
\hline \multicolumn{8}{|l|}{ MNV type, $n(\%)$} \\
\hline Type 1 & $54 \%$ & $54 \%$ & $54 \%$ & $57 \%$ & $55 \%$ & $55 \%$ & $52 \%$ \\
\hline Type 2 & $20 \%$ & $17 \%$ & $16 \%$ & $21 \%$ & $23 \%$ & $22 \%$ & $20 \%$ \\
\hline Type 3 & $5 \%$ & $0 \%$ & $4 \%$ & $5 \%$ & $4 \%$ & $8 \%$ & $7 \%$ \\
\hline Other $^{a}$ & $7 \%$ & $8 \%$ & $9 \%$ & $6 \%$ & $5 \%$ & $4 \%$ & $7 \%$ \\
\hline Unknown & $14 \%$ & $21 \%$ & $17 \%$ & $12 \%$ & $13 \%$ & $11 \%$ & $14 \%$ \\
\hline \multicolumn{8}{|l|}{ Baseline injection type, $n$ (\%) } \\
\hline Bevacizumab & $431(28 \%)$ & $72(45 \%)$ & $81(32 \%)$ & $83(27 \%)$ & $65(25 \%)$ & $50(20 \%)$ & $80(26 \%)$ \\
\hline Aflibercept & $509(33 \%)$ & $37(23 \%)$ & $74(29 \%)$ & $97(32 \%)$ & $94(36 \%)$ & $100(40 \%)$ & $107(35 \%)$ \\
\hline Ranibicumab & $598(39 \%)$ & $51(32 \%)$ & $99(39 \%)$ & $127(41 \%)$ & $103(39 \%)$ & $100(40 \%)$ & $118(39 \%)$ \\
\hline Baseline VA, mean (SD) & $60.3(17.7)$ & $62.1(16.8)$ & $61.3(16.7)$ & $60.6(17.6)$ & $60.5(18.3)$ & $59.7(18.5)$ & $58.7(18)$ \\
\hline$\leq 35$ letters, $n$ (\%) & $170(11 \%)$ & $13(8 \%)$ & $23(9 \%)$ & $34(11 \%)$ & $32(12 \%)$ & $27(11 \%)$ & $41(13 \%)$ \\
\hline$\geq 70$ letters, $n$ (\%) & $571(37 \%)$ & $68(42 \%)$ & $94(37 \%)$ & $122(40 \%)$ & $106(40 \%)$ & $82(33 \%)$ & $99(32 \%)$ \\
\hline \multicolumn{8}{|l|}{ Injection type 24 months, $n$ (\%) } \\
\hline Bevacizumab & $264(17 \%)$ & $41(26 \%)$ & $51(20 \%)$ & $48(16 \%)$ & $38(15 \%)$ & $37(15 \%)$ & $49(16 \%)$ \\
\hline Aflibercept & $801(52 \%)$ & $83(52 \%)$ & $130(51 \%)$ & $160(52 \%)$ & $141(54 \%)$ & $130(52 \%)$ & $157(51 \%)$ \\
\hline Ranibicumab & $473(31 \%)$ & $36(22 \%)$ & $73(29 \%)$ & $99(32 \%)$ & $83(32 \%)$ & $83(33 \%)$ & $99(32 \%)$ \\
\hline VA 24 months, mean (SD) & $64.4(19.6)$ & $64.9(17.1)$ & $66.2(17.1)$ & $65.9(18.6)$ & $64.3(20.6)$ & $63.7(20.6)$ & $61.7(21.5)$ \\
\hline$\leq 35$ letters, $n$ (\%) & $169(11 \%)$ & $18(11 \%)$ & $19(7 \%)$ & $30(10 \%)$ & $31(12 \%)$ & $28(11 \%)$ & $43(14 \%)$ \\
\hline$\geq 70$ letters, $n$ (\%) & $857(56 \%)$ & $83(52 \%)$ & $149(59 \%)$ & $191(62 \%)$ & $145(55 \%)$ & $131(52 \%)$ & $158(52 \%)$ \\
\hline$\triangle V A$, mean $(95 \% \mathrm{Cl})$ & $4(3.2,4.9)$ & $2.8(-0.1,5.7)$ & $4.9(2.9,6.9)$ & $5.2(3.2,7.2)$ & $3.8(1.6,6)$ & $4(2.1,5.9)$ & $3(0.8,5.2)$ \\
\hline Active MNV 24 months, \% visits & $57 \%$ & $75 \%$ & $66 \%$ & $60 \%$ & $52 \%$ & $44 \%$ & $51 \%$ \\
\hline
\end{tabular}

CI Confidence interval, MNV Macular neovascular, SD Standard deviation, VA Visual acuity.

${ }^{a}$ Other includes juxtapapillary and polypoidal choroidal vasculopathy.

treated at $>12$-week intervals (58.7 letters) and only a 3-letter difference with the shortest treatment intervals (global $P=0.388$ ).

Similarly, the mean VA of the groups at 2 years was similar (61.7 letters for $>12$-week group, 63.7 letters for the 12 week group, 64.3 letters at the 10 week group, 65.9 letters at the 8 weeks group, 66.2 letters for the 6-week group and 64.9 letters for the 4-week group $(P=0.08))$. The proportion of visits with active MNV over the first 2 years, however, was significantly different amongst treatment interval groups, showing a clear trend of increase from the long interval to short interval groups from $44 \%$ of visits in the 12 -week group to $75 \%$ in the 4 -week group, although the $>12$ week group had a higher proportion of active visits than the 12week group (52\%; global $P<0.001)$.

\section{Rate of non-persistence after the first $\mathbf{2 4}$ months of treatment} The overall rate of non-persistence was $43 \%(n=659)$ from the first 24 months to 5 years. When divided by treatment interval groups, significantly more eyes were found to be non-persistent in the longer versus the shorter treatment interval groups $(P<0.001$, Fig. 2).

The relationship between various factors and subsequent non-persistence after multivariate adjustment is summarized in Table 2. A longer treatment interval at 2-years was significantly associated with subsequent non-persistence, with eyes with treatment intervals of 12 or $>12$ weeks having $53 \%$ higher risk of non-persistence than eyes with treatment intervals of 4 weeks (HR 1.53, $P=0.002$ ). In addition, younger patients and those with better visual acuity at 2 years were less likely to be nonpersistent independent of their treatment intervals at 2 years. The hazards ratios for risk of non-persistence are summarised in Table 2.

Sensitivity analyses using the average injection frequency over 2 years yielded similar results (Table S1 and Fig. S1 in Supplementary Material). Eyes that received more injections (i.e., shorter average treatment intervals) had the lowest rate of nonpersistence while those receiving fewer injections had greater rates of non-persistence $(P=0.004)$.

Reasons for discontinuation from the study were provided in 210 of 781 eyes. These included 55 patient deaths, 62 patients with further treatment deemed futile, 3 patients with medical contraindications, 26 patient who declined further treatments, 36 patients visited another doctor, and 28 patients with successful treatments with the remaining 571 eyes unaccounted for. The reasons partitioned by treatment interval category are provided in Table S2 in the Supplementary materials.

\section{Clinical outcomes at 5 years}

There was a mean $(95 \% \mathrm{Cl})$ loss of $-0.6(-2.1,0.1)$ letters from baseline to 5 years in the entire cohort that completed 5 years of follow up. The outcomes for this 5 year completer cohort, stratified by treatment interval group, are reported in Table 3 . The change in VA from baseline to 5 years varied across the treatment interval groups. The 12-week group had the largest loss with a -4.4 letters, followed by the 4-week group with a loss of -2.3 letters. 


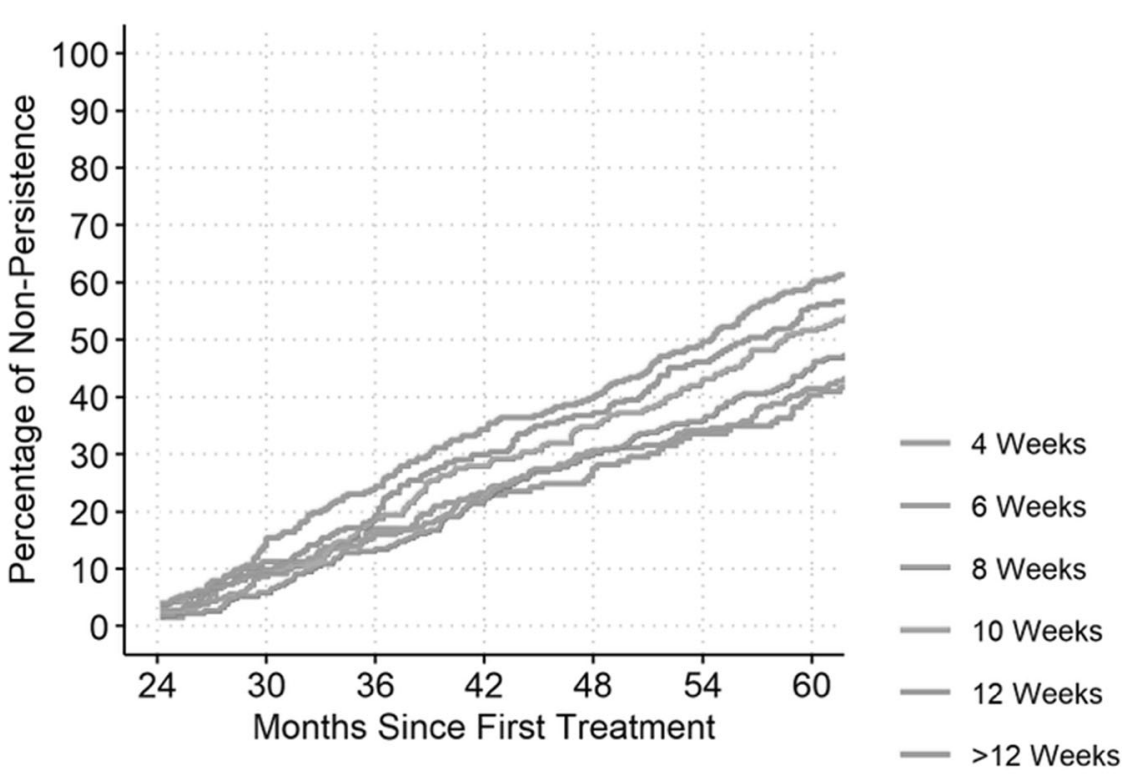

Number at risk

$\begin{array}{ccccccc}160 & 141 & 128 & 118 & 109 & 98 & 88 \\ 254 & 228 & 207 & 188 & 165 & 155 & 134 \\ 307 & 286 & 262 & 233 & 206 & 183 & 155 \\ 262 & 230 & 205 & 178 & 159 & 135 & 113 \\ 250 & 212 & 185 & 154 & 135 & 113 & 92 \\ 305 & 248 & 216 & 184 & 163 & 136 & 106\end{array}$

Fig. 2 Kaplan-Meier survival curve of time until non-persistence by average treatment interval over the three visits prior to and including the 2-year visit. The survival curve begins at 24 months as a minimum of 24 months of follow-up was required to be eligible for analysis, however, some eyes may have dropped out immediately thereafter. The number at risk are displayed below the plot.

Table 2. Hazards ratios for risk of non-persistence to long-term treatment estimated from Cox-proportional hazards models. Cox models included adjustment for nesting of outcomes within patients treated in both eyes and patients treated by the same clinician. Significant $p$-values are indicated in bold.

\begin{tabular}{|c|c|c|}
\hline Variable (reference group) & Hazards ratio $(95 \% \mathrm{Cl})$ & $P$-value \\
\hline Gender (female) & $1.06(0.91,1.25)$ & 0.445 \\
\hline Age, per year & $1.02(1.01,1.04)$ & $<0.001$ \\
\hline VA 24 months, per 5 letters & $0.95(0.93,0.97)$ & $<0.001$ \\
\hline \multicolumn{3}{|l|}{ Type 1 MNV } \\
\hline Type 2 MNV & $1.11(0.91,1.36)$ & 0.593 \\
\hline Type 3 MNV & $1.02(0.72,1.44)$ & \\
\hline Other & $1.19(0.87,1.63)$ & \\
\hline \multicolumn{3}{|c|}{ Injection type 24 months (Bevacizumab) } \\
\hline Aflibercept & $0.90(0.62,1.32)$ & 0.334 \\
\hline Ranibizumab & $1.08(0.73,1.60)$ & \\
\hline \multicolumn{3}{|c|}{ Treatment interval group (4-weeks) } \\
\hline 6 weeks & $1.02(0.75,1.39)$ & 0.002 \\
\hline 8 weeks & $1.00(0.74,1.34)$ & \\
\hline 10 weeks & $1.05(0.77,1.44)$ & \\
\hline 12 weeks & $1.32(0.96,1.82)$ & \\
\hline$>12$ weeks & $1.53(1.14,2.04)$ & \\
\hline
\end{tabular}

Bold values indicate statistical significance $p<0.05$.
The 6-week group had the best outcomes with a gain of 1.6 letters. Only the change in VA from baseline to 5 years in the 12week group was significant $(P=0.027)$. Shorter treatment intervals at 2 years also corresponded to more injections and higher levels of activity throughout the 5-year study period. The disease activity over time was also activity remained highest in the shorter treatment interval groups throughout the entire 60-month study period.

\section{DISCUSSION}

In this analysis we found no evidence that longer treatment intervals were more compliant with treatment, in. fact we found the reverse. Patients receiving less frequent treatment at 2 years in the present analysis were more likely to be non-persistent, which occurred in about half the cohort, up to 5 years. Our findings are consistent with other studies that have found that treatment burden is not a key driver of non-persistence, with only $8-19 \%$ of all cases citing treatment burden as a reason for non-persistence or non-adherence to treatment $[6,17]$.

We suggest several reasons for this observation. Our data show that higher treatment frequency at shorter intervals was also associated with more disease activity. Many these patients likely experience a drop in vision immediately prior to their treatments and subsequent improvement, hence continue to be motivated to receive regular treatments for fear of losing vision. Patients have been reported to perceive treatment as necessary, even if administered monthly, and were willing to tolerate it if positive visual outcomes could be expected $[18,19]$. Up to $73 \%$ of patients in one study were influenced by a change in visual acuity 


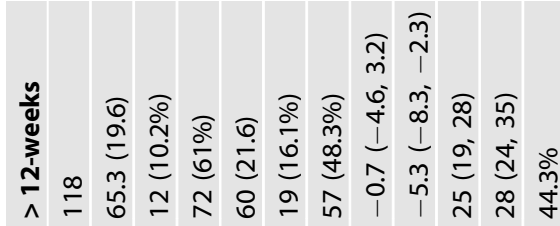

โุ?
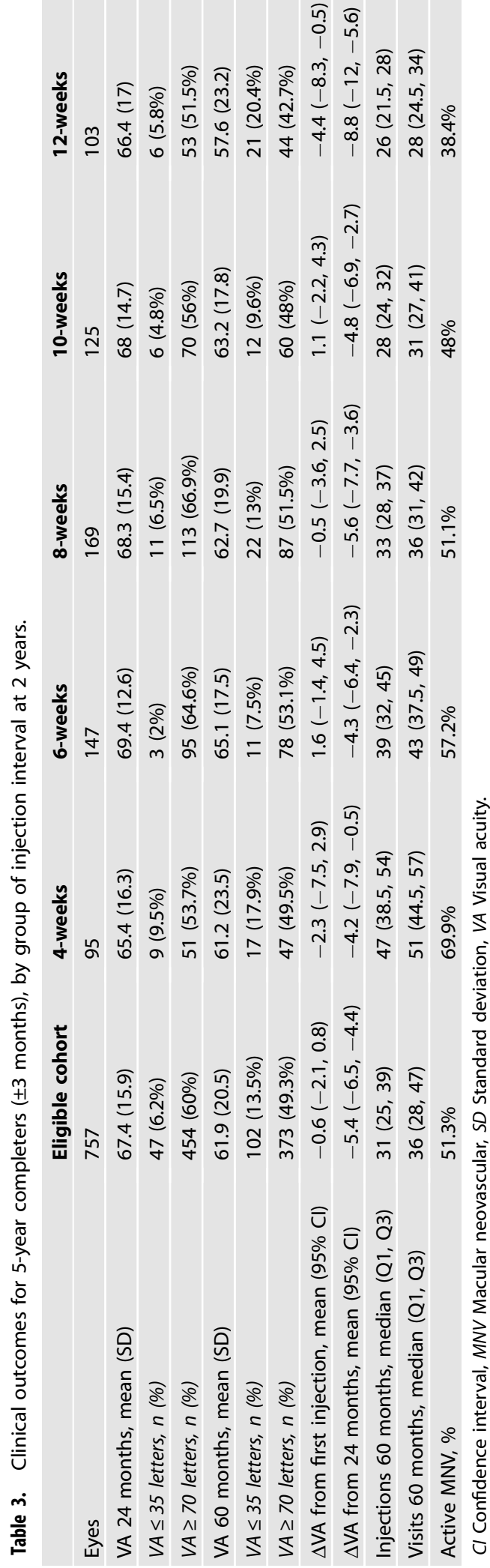

outcomes when it came to tolerating treatments and they were also willing to accept additional time spent per visit (including travel, waiting, and treatment time) for VA improvement [20]. Patients have also been reported to be more compliant to treatment regimens that allowed for better planning of appointments (fixed or treat and extend) rather than regimens that had monitoring visits (PRN) [5].

Conversely, patients treated on longer intervals at 24 months were likely to be non-persistent later in their treatment journey. This may be because they have become complacent with their treatments due to longer treatment intervals, lower disease activity and lack of a subjective improvement with treatment that will no longer occur once the lesion has become inactive. This is supported by our data where patients with longer treatment intervals had correspondingly fewer active visits. A previous study suggested that non adherence was due to complacence with treatment as $40 \%$ of these patients were found to have recurrent disease after a period of disease quiescence in the first 12 months of treatment [9]. Non persistence to treatment is risky with studies reporting the disease recurrence rate of up to $50 \%$ in the first 12 months from the cessation of treatment [21]. Vision may be rescued in cases of prompt retreatment for early recurrence of disease but there is significant long term detriment to vision with recurrence of disease. Recurrence of disease has been shown to result in irreversible vision loss in the second year data from the VEGF Trap-Eye: Investigation of Efficacy and Safety in Wet AgeRelated Macular Degeneration (VIEW) and Lucentis Compared to Avastin Study (LUCAS) trials [22, 23]. A large, long term real world study also reported $41 \%$ reactivating in the first year after suspending treatment in eyes with 3 month treatment intervals and $79 \%$ at 5 years. Eyes that reactivated also loss 4.3 letters and only managed to recover 1.2 letters with the recommencement of treatment [24].

In this analysis we attempted to examine treatment burden as the main factor of association with long term non-persistence. A major confounder is the effect of treatment futility on nonpersistence. Several aspects of the methodology and results help to correct for this. Firstly, treatment interval at 2 years was used to ensure that the cohort studied were still receiving treatment at that time or would not have been included in this analysis. Secondly, baseline and final VA at 2 years was similar across all groups suggesting that all patients were treated as necessary to achieve their best possible outcomes. Another possible confounder is the disruption to ophthalmic services and subsequent lockdowns due to the COVID-19 pandemic in 2020 which might have increased non-persistence rates. However, this would only have affected the 5-year non-persistence of patients that started treatment in 2015 and were still continuing treatment in 2020. Since we did not investigate non-persistence rates beyond 5 years, we believe any disruption caused by COVID-19 would be negligible in our cohort.

The reasons for non-persistence are multi-factorial. While treatment interval at 2-year had a strong effect on nonpersistence, other factors identified in a prior study include patient-related factors such as increased comorbidities, lack of carer assistance, longer distance from home to clinics and poorer baseline visual acuity. On the other hand, patients who experienced greater visual gains have been reported to be more likely to persist with treatment [25]. We believe that this also applies to our cohort.

A strength of this study is the analysis of a large multi-centred cohort of patients with outcome data that were prospectively collected. All data analysed by FRB! are $100 \%$ complete and within prespecified ranges hence, we believe that the results presented are robust and representative of routine clinical practice. This study also addressed a specific and clinically important question, the effect of treatment burden on long-term non-persistence, that may be difficult to answer with any other study design. A 
significant limitation of this study was the lack of data on the reasons for non-persistence for most of our patient cohort. Discontinuation might have been due to futility of treatment, if this were the case we would expect to find that patients that discontinued had worse vision but in fact this was not the case.

Overall, these findings suggest that patients are able to tolerate a high treatment burden if they get better outcomes. These findings should encourage physicians to continue to treat patients in accordance to their disease activity without worry that patients will'burn-out'. In addition, awareness and education of the disease is important in patients with more stable disease to ensure that they are not lulled into a sense of complacency once the underlying neovascular lesion has been deactivated. A modification to the treat and extend regimen should include a final step of maintain and remind patients and physicians regarding the need for regular treatment or at the very least timely monitoring.

\section{Summary table}

What was known before

- Early treatment burden related to neovascular age related macular degeneration (nAMD) may affect long term persistence to treatment Persistence is defined as continuing treatment Shorter treatment intervals, hence higher treatment burden is thought to contribute to reduced persistence to treatment

\section{What this study adds}

- Contrary to prior findings, we found that patients on longer treatment intervals at 2 years were more likely to be nonpersistent with treatment in later years This suggest that physicians should treat patients according to disease activity and not be apprehensive of a high treatment burden Patients appear to value improvement in vision and hence persist with treatment even in the long term.

\section{REFERENCES}

1. Cohen SY, Mimoun G, Oubraham H, Zourdani A, Malbrel C, Quere S, et al. Changes in visual acuity in patients with wet age-related macular degeneration treated with intravitreal ranibizumab in daily clinical practice: The LUMIERE study. Retina. 2013;33:474-81.

2. Gillies MC, Walton RJ, Arnold JJ, McAllister IL, Simpson JM, Hunyor AP, et al. Comparison of outcomes from a phase 3 study of age-related macular degeneration with a matched, observational cohort. Ophthalmology. 2014;121:676-81.

3. Rakic JM, Leys A, Brie H, Denhaerynck K, Pacheco C, Vancayzeele S, et al. Realworld variability in ranibizumab treatment and associated clinical, quality of life, and safety outcomes over 24 months in patients with neovascular age-related macular degeneration: The HELIOS study. Clin Ophthalmol. 2013;7:1849-58.

4. Rao P, Lum F, Wood K, Salman C, Burugapalli B, Hall R, et al. Real-world vision in age-related macular degeneration patients treated with single Anti-VEGF drug type for 1 year in the IRIS Registry. Ophthalmology. 2018;125:522-8.

5. Ehlken C, Ziemssen F, Eter N, Lanzl I, Kaymak H, Lommatzsch A, et al. Systematic review: Non-adherence and non-persistence in intravitreal treatment. Graefes Arch Clin Exp Ophthalmol. 2020;258:2077-90.

6. Okada M, Mitchell P, Finger RP, Eldem B, Talks SJ, Hirst C, et al. Nonadherence or nonpersistence to intravitreal injection therapy for neovascular age-related macular degeneration: A mixed-methods systematic review. Ophthalmology. 2021;128:234-47.

7. Adrean SD, Chaili S, Ramkumar H, Pirouz A, Grant S. Consistent long-term therapy of neovascular age-related macular degeneration managed by 50 or more antiVEGF injections using a treat-extend-stop protocol. Ophthalmology. 2018;125:1047-53.

8. Barthelmes D, Nguyen V, Daien V, Campain A, Walton R, Guymer R, et al. Two year outcomes of "Treat and extend" intravitreal therapy using aflibercept preferentially for neovascular age-related macular degeneration. Retina. 2018;38:20-8.
9. Chong Teo KY, Saxena N, Gan A, Wong TY, Gillies MC, Chakravarthy U, et al. Detrimental effect of delayed re-treatment of active disease on outcomes in neovascular age-related macular degeneration: The RAMPS study. Ophthalmol Retin. 2020;4:871-80.

10. Essex RW, Nguyen V, Walton R, Arnold JJ, McAllister IL, Guymer RH, et al. Treatment patterns and visual outcomes during the maintenance phase of treat-andextend therapy for age-related macular degeneration. Ophthalmology. 2016;123:2393-400.

11. Gillies MC, Campain A, Barthelmes D, Simpson JM, Arnold JJ, Guymer RH, et al. Long-term outcomes of treatment of neovascular age-related macular degeneration: Data from an observational study. Ophthalmology. 2015;122:1837-45.

12. Schmidt-Erfurth $U$, Chong V, Loewenstein A, Larsen M, Souied E, Schlingemann R, et al. Guidelines for the management of neovascular age-related macular degeneration by the European Society of Retina Specialists (EURETINA). Br J Ophthalmol. 2014;98:1144-67.

13. Silva R, Berta A, Larsen M, Macfadden W, Feller C, Mones J, et al. Treat-and-extend versus monthly regimen in neovascular age-related macular degeneration: Results with Ranibizumab from the TREND study. Ophthalmology. 2018;125:57-65.

14. Wykoff CC, Croft DE, Brown DM, Wang R, Payne JF, Clark L, et al. Prospective trial of treat-and-extend versus monthly dosing for neovascular age-related macular degeneration: TREX-AMD 1-year results. Ophthalmology. 2015;122:2514-22.

15. Gillies MC, Walton R, Liong J, Arnold JJ, McAllister I, Morlet N, et al. Efficient capture of high-quality data on outcomes of treatment for macular diseases: The fight retinal blindness! Project. Retina. 2014;34:188-95.

16. Barthelmes D, Walton R, Campain AE, Simpson JM, Arnold JJ, McAllister IL, et al. Outcomes of persistently active neovascular age-related macular degeneration treated with VEGF inhibitors: observational study data. $\mathrm{Br} J$ Ophthalmol. 2015;99:359-64.

17. Mones J, Singh RP, Bandello F, Souied E, Liu X, Gale R. Undertreatment of neovascular age-related macular degeneration after 10 years of anti-vascular endothelial growth factor therapy in the real world: The need for a change of mindset. Ophthalmologica. 2020;243:1-8.

18. Boyle J, Vukicevic M, Koklanis K, Itsiopoulos C. Experiences of patients undergoing anti-VEGF treatment for neovascular age-related macular degeneration: $A$ systematic review. Psychol Health Med. 2015;20:296-310.

19. Boyle J, Vukicevic M, Koklanis K, Itsiopoulos C, Rees G. Experiences of patients undergoing repeated intravitreal anti-vascular endothelial growth factor injections for neovascular age-related macular degeneration. Psychol Health Med. 2018;23:127-40.

20. Droege KM, Muether PS, Hermann MM, Caramoy A, Viebahn U, Kirchhof B, et al. Adherence to ranibizumab treatment for neovascular age-related macular degeneration in real life. Graefes Arch Clin Exp Ophthalmol. 2013;251:1281-4.

21. Aslanis S, Amren U, Lindberg C, Epstein D. Recurrent neovascular age-related macular degeneration after discontinuation of vascular endothelial growth factor inhibitors managed in a treat-and-extend regimen. Ophthalmol Retina. 2021;6:15-20.

22. Richard G, Mones J, Wolf S, Korobelnik JF, Guymer R, Goldstein M, et al. Scheduled versus pro re nata dosing in the VIEW trials. Ophthalmology. 2015;122:2497-503.

23. Berg K, Hadzalic E, Gjertsen I, Forsaa V, Berger LH, Kinge B, et al. Ranibizumab or Bevacizumab for neovascular age-related macular degeneration according to the lucentis compared to avastin study treat-and-extend protocol: Two-year results. Ophthalmology. 2016;123:51-9.

24. Nguyen V, Vaze A, Fraser-Bell S, Arnold J, Essex RW, Barthelmes D, et al. Outcomes of suspending VEGF inhibitors for neovascular age-related macular degeneration when lesions have been inactive for 3 months. Ophthalmol Retin. 2019;3:623-8.

25. Okada M, Kandasamy R, Chong EW, McGuiness M, Guymer RH. The treat-andextend injection regimen versus alternate dosing strategies in age-related macular degeneration: A systematic review and meta-analysis. Am J Ophthalmol. 2018;192:184-97.

\section{ACKNOWLEDGEMENTS}

Fight Retinal Blindness! investigators: Auckland District Health Board, New Zealand (Dr D Squirrell); Amphia Ziekenhuis, Breda, Netherlands (Ms C Brooijmans, Dr O Tigchelaar-Besling); Armadale Eye Clinic, Victoria (Dr A Cohn); Auckland Eye, New Zealand (Dr A McGeorge); Bergman Clinics, Velp, Netherlands (Dr P Peters); Bundaberg Eye Clinic, Queensland (Dr I McLean); Cairns Eye Surgery, Queensland (Dr A Field); Camberwell Retina Specialists, Victoria (Dr S Wickremasinghe); Canberra Hospital, Australian Capital Territory (Dr C Dayajeewa, Dr R Essex, Dr J Wells); Care Foresight, New South Wales (Dr A Dunlop); Central Coast Eye Specialist, New South Wales (Dr S Young); Centre for Eye Research Australia, Victoria (Professor R Guymer); Centro de Ojos de La Coruña, Spain (Dr P Carnota, Dr C Torres Borrego); Clinica Oftalvist Valencia, Spain (Dr R Gallego-Pinazo); Coastwide Eye Surgery, New South 
Wales (Dr R Ferrier); Crest Eye Associates, New Zealand (Dr J Ah-Chan); Doncaster Eye Center, Victoria (Dr L Chow); Dr Alex Amini's Practice, Victoria (Dr A Amini); Dr Clarks Practice, New South Wales (Dr G Clark); Dr Nadia Wittles Practice, South Australia (Dr N Wittles); Dr. Phillip Windle, Queensland (Dr P Windle); Dr. Russell Phillips Practice, South Australia (Dr R Phillips); Erasmus Medical Center - Dept Ophthalmology, Netherlands (Professor J Vingerling); Eye Associates, New South Wales (Dr M Gillies, Dr A Hunt); Eye Doctors Mona Vale, New South Wales (Dr P Beaumont); Eye Specialists Greensborough, Victoria (Dr L Chow); Eye Surgeons Miranda, New South Wales (Dr A Hunt); Eyemedics (Wayville), South Australia (Dr K Billing, Dr J Chen, Dr S Lake, Dr J Landers, Dr M Perks, Dr D Qatarneh, Dr N Saha); Fundació Privada Hospital Asil de Granollers, Spain (Dr L Sararols); Fondazione IRCCS Ca' Granda Ospedale Maggiore Policlinico, Italy (Dr F Viola); Gladesville Eye Specialists, New South Wales (Dr S Young); Hospital Universitario Principe de Asturias, Spain (Dr R Montejano Milner, Mrs C Arruabarrena); Hawthorn Eye Clinic, Victoria (Dr L Chow); Hornsby Eye Specialists, New South Wales (Dr S Lal); Hospital Clinico Universitario Lozano Blesa, Spain (Dr A Boned Murillo, Mr G Perez Rivases); Hospital Clínic de Barcelona, Spain (Ms S Alforja Castiella, Dr R Casaroli-Marano, Dr M Figueras-Roca); Hospital Costa del Sol, Spain (Dr A Chinchurreta Capote); Hospital Tor Vergata Roma, Italy (Professor F Ricci); Hospital Universitario Fundacion Jimenez Diaz, Spain (Dr E Carreño, Mrs N Munoz Sanz, Mr N Ventura Abreu); Hospital Universitario La Paz, Spain (Dr M Asencio Duran); Hospital Universitario Miguel Servet, Spain (Dr P Calvo, Dr J Sanchez); Hospital Universitario Puerta de Hierro, Spain ( $\mathrm{Dr}$ I Flores-Moreno, Mrs M Garcia Zamora); Hospital Universitario Virgen del Rocio, Spain ( $\mathrm{Dr} M$ Rodriguez Maqueda); Hospital Universitario del Henares, Spain (Dr A Fernández Hortelano); Isala, Zwolle, Netherlands (Dr D Ponsioen); Les Manning, Queensland (Dr L Manning); Luigi Sacco Hospital - University of Milan, Italy (Dr A Invernizzi); Maison rouge Ophthalmologic center, France (Dr L Castelnovo); Marsden Eye Specialists, New South Wales (Dr J Arnold); Mater Private Hospital, Ireland (Dr L O'Toole); Midwest Ophthalmology, New South Wales (Dr K Tang); Mona Vale Eye Centre, New South Wales (Dr C Chung); Montpellier CHU, France (Professor V Daien); Nepean Valley Eye Surgeons, New South Wales (Dr G Banerjee); Oogziekenhuis AMC Zonnestraal, Netherlands (Dr F Verbraak); Oogziekenhuis Zonnestraal - Hilversum, Netherlands (Dr F Verbraak); Park Street Eye Clinic Tauranga, New Zealand (Dr A Thompson); Port Macquarie Eye Centre, New South Wales (Dr J Game, Dr C Thompson); Retina \& Macula Specialists (Miranda), New South Wales (Dr M Chilov, Dr S Nothling); Retina Associates, New South Wales (Dr S FraserBell, Dr A Fung, Dr C Younan); Retina Specialists, New Zealand (Dr R Barnes, Dr A Vincent); Rotorua Eye Clinic, New Zealand (Dr N Murray); Singapore National Eye Centre, Singapore (Dr G Cheung); Specialist Eye Group, Victoria (Dr L Chow, Dr A Cohn); St. Elisabeth ziekenhuis, Tilburg, Netherlands (Dr M van Hecke); Strathfield Retina Clinic, New South Wales (Dr C Chung, Dr J Wong); Sydney Eye Hospital, New South Wales (Dr R Chong, Dr S Fraser-Bell, Dr M Gillies); Tamworth Eye Centre, New South Wales (Dr P Hinchcliffe); USZ AUG, Switzerland (Dr D Barthelmes); Unidad de Gestion Clinica de Oftalmologia, Hospital de Txagorritxu, Spain (Dr E Diaz De Durana
Santa Coloma, Dr G Garay-Aramburu); Vall de Hebron University Hospital, Spain (Dr H Brosa Morros); Victoria Parade Eye Consultants, Victoria (Dr M Daniell, Professor R Guymer, Dr A Harper, Dr J O’Day); Victorian Eye Surgeons, Victoria (Dr A Cohn); Visionary Eye Specialists, New South Wales (Dr C Hooper).

\section{AUTHOR CONTRIBUTIONS}

KYCT, VN, and MCG conceived the study. KYCT, VN, and MCG planned the study. KYCT, LO, VD, JSM, FR, TLP, HBM, CMGC, JJA, DB, and MCG were involved in data collection. $K Y C T, V N$, and $M C G$ undertook data analysis and interpretation. $K Y C T, V N$, CMGC, and MCG wrote the paper. LO, VD, JSM, FR, TLP, HBM, JJA, and DB reviewed and added to the final paper.

\section{FUNDING}

Supported by a grant from the Royal Australian NZ College of Ophthalmologists Eye Foundation (2007-2009), a grant from the National Health and Medical Research Council, Australia (NHMRC 2010-2012), and a grant from the Macula Disease Foundation, Australia. Mark Gillies is a Sydney Medical Foundation Fellow and is supported by an NHMRC practitioner fellowship. Daniel Barthelmes was supported by the Walter and Gertrud Siegenthaler Foundation Zurich, Switzerland, and the Swiss National Foundation. Kelvin Teo and Gemmy Cheung are supported grants by a grant from the National Medical Research Council, (Open Fund Large Collaborative grant no: NMRC/LCG/0042018 and TA19nov-0002). Funding was also provided by Novartis. Novartis made non-binding comments on the design of the study.

\section{COMPETING INTERESTS}

The authors declare no competing interests.

\section{ADDITIONAL INFORMATION}

Supplementary information The online version contains supplementary material available at https://doi.org/10.1038/s41433-022-01957-z.

Correspondence and requests for materials should be addressed to Kelvin Y. C. Teo.

Reprints and permission information is available at http://www.nature.com/ reprints

Publisher's note Springer Nature remains neutral with regard to jurisdictional claims in published maps and institutional affiliations. 